\title{
Novel biomarker identification using metabolomic profiling to differentiate radiation necrosis and recurrent tumor following Gamma Knife radiosurgery
}

\author{
*Alex Y. Lu, BA, ${ }^{1}$ Jack L. Turban, BA, ${ }^{1}$ Eyiyemisi C. Damisah, MD, ${ }^{1} \mathrm{Jie} \mathrm{Li}, \mathrm{MD}, \mathrm{PhD},{ }^{2}$ \\ Ahmed K. Alomari, MD, ${ }^{2}$ Tore Eid, MD, PhD, ${ }^{3}$ Alexander O. Vortmeyer, MD, PhD, ${ }^{2}$ and \\ Veronica L. Chiang, MD' \\ Departments of ${ }^{1}$ Neurosurgery, ${ }^{2}$ Pathology, and ${ }^{3}$ Laboratory Medicine, Yale School of Medicine, New Haven, Connecticut
}

OBJECTIVE Following an initial response of brain metastases to Gamma Knife radiosurgery, regrowth of the enhancing lesion as detected on MRI may represent either radiation necrosis (a treatment-related inflammatory change) or recurrent tumor. Differentiation of radiation necrosis from tumor is vital for management decision making but remains difficult by imaging alone. In this study, gas chromatography with time-of-flight mass spectrometry (GC-TOF) was used to identify differential metabolite profiles of the 2 tissue types obtained by surgical biopsy to find potential targets for noninvasive imaging.

METHODS Specimens of pure radiation necrosis and pure tumor obtained from patient brain biopsies were flash-frozen and validated histologically. These formalin-free tissue samples were then analyzed using GC-TOF. The metabolite profiles of radiation necrosis and tumor samples were compared using multivariate and univariate statistical analysis. Statistical significance was defined as $p \leq 0.05$.

RESULTS For the metabolic profiling, GC-TOF was performed on 7 samples of radiation necrosis and 7 samples of tumor. Of the 141 metabolites identified, 17 (12.1\%) were found to be statistically significantly different between comparison groups. Of these metabolites, 6 were increased in tumor, and 11 were increased in radiation necrosis. An unsupervised hierarchical clustering analysis found that tumor had elevated levels of metabolites associated with energy metabolism, whereas radiation necrosis had elevated levels of metabolites that were fatty acids and antioxidants/cofactors.

CONCLUSIONS To the authors' knowledge, this is the first tissue-based metabolomics study of radiation necrosis and tumor. Radiation necrosis and recurrent tumor following Gamma Knife radiosurgery for brain metastases have unique metabolite profiles that may be targeted in the future to develop noninvasive metabolic imaging techniques.

https://thejns.org/doi/abs/10.3171/2016.8.JNS161395

KEY WORDS radiation necrosis; Gamma Knife radiosurgery; brain metastases; metabolomics; mass spectrometry; stereotactic radiosurgery

$\mathrm{G}$ AMma knife radiosurgery (GKS) is a highly effective treatment for brain metastases, especially when the tumors are multiple, small, and surgically less accessible. The highly conformal delivery of radiation to the metastases results in relatively little radiation exposure to the surrounding brain. In a minority of cases, following an initial good response to GKS treatment, the tumor being monitored using MRI can start to regrow approxi- mately 12 months after initial therapy. Radiographically, this appears as regrowth of the gadolinium-enhancing lesion on T1-weighted imaging associated with an increase in the amount of surrounding FLAIR signal. Histological examination of these regrowing lesions, however, can show either recurrent tumor or a treatment-related inflammatory process known as radiation necrosis. Radiation necrosis can be identified on histological examination by a central

ABBREVIATIONS AUC = area under the curve; GC-TOF = gas chromatography with time-of-flight mass spectrometry; GKS = Gamma Knife radiosurgery; MRS = MR spectroscopy; NAA = N-acetylaspartate; NSCLC $=$ non-small cell lung cancer; $\mathrm{OCT}=$ optimal cutting temperature; ROC $=$ receiver operating characteristic. SUBMITTED May 31, 2016. ACCEPTED August 17, 2016.

INCLUDE WHEN CITING Published online November 25, 2016; DOI: 10.3171/2016.8.JNS161395.

* Mr. Lu and Mr. Turban contributed equally to this work. 
zone of coagulation necrosis surrounded by inflammatory demyelination, astrocytosis, vascular hyalinization, and reactive edema. ${ }^{1,12}$

Despite clear histological differences and extensive research in the area, distinguishing radiation necrosis from recurrent tumor using noninvasive tests remains difficult. Currently available techniques for trying to differentiate the 2 entities include MR spectroscopy (MRS), MR perfusion, MR diffusion, and PET imaging with tracers such as methionine-PET. Although diagnostic accuracy rates of up to $80 \%$ have been reported at dedicated centers, the differentiation remains far from perfect when used in standard clinical practice.

Differentiating the 2 processes, however, is vital to patient management decision making. Recurrent tumor may warrant further radiation treatment, whereas radiation necrosis is typically managed with corticosteroids and other medical or surgical approaches because re-irradiation may worsen clinical status. To make the correct diagnosis, the current standard of care for these patients is to undergo invasive brain biopsy, ${ }^{9}$ exposing them to surgical and perioperative risks associated with considerable health care costs.

Given that radiation necrosis and tumor are distinct cellular processes, it would be expected that their metabolite profiles should also be distinguishable. Metabolomic profiling using gas chromatography with time-of-flight mass spectrometry (GC-TOF) analysis allows investigators to evaluate a diverse range of low-molecular-weight metabolites. ${ }^{7,10,17}$ In this study, we implemented GC-TOF on flash-frozen biopsy-confirmed radiation necrosis and tumor samples following GKS to identify differential metabolite levels between the comparison groups.

\section{Methods}

\section{Patient Cohort}

All patients undergoing GKRS at our institution signed consent for the details of their radiosurgery treatment and subsequent clinical course to be entered prospectively into a database. The names of patients who had to undergo resection following radiosurgery were retrieved retrospectively, and any available tissue from these patients was retrieved from pathology archives for this project.

\section{Collection and Pathological Examination of Tissue Samples}

All patients in this study had brain metastases that were treated with GKS at the Yale Gamma Knife Center using the Leksell Gamma Knife 4C machine; radiosurgery dosing was based on lesion volume as guided by RTOG90-05. At the time of lesional regrowth, as seen by gadolinium-enhanced MRI on follow-up imaging, each case was discussed at a multidisciplinary tumor board and each patient was selected to undergo resection of their regrowing lesions.

Immediately after surgical excision, all resected samples were bisected and processed in 2 ways. Half of the sample was processed into formalin and paraffin, whereas the other half was snap-frozen in optimal cutting temperature (OCT) compound. For each sample, sections from the paraffin block and frozen sections from the OCT block were concurrently examined histologically. Given that imaging changes after radiation can represent tumor regrowth, radiation injury, or a combination of both, each specimen intended for metabolomic analysis was analyzed histologically first.

Pure areas of radiation necrosis and pure areas of tumor were then separated from the resected specimens by a neuropathologist (A.O.V.), as shown in Fig. 1. On histological examination, radiation necrosis was defined as 3 concentric rings of tissue with innermost necrosis, a middle region of reactive gliosis with demyelination, and an outer ring of edema. As the distinguishing pathology, the middle region of reactive gliosis in the absence of tumor cells was separated from the other regions and sent for analysis after being matched to tumor sample of the same primary tumor type.

Upon dividing the surgical samples into either radiation necrosis or tumor, tissue samples were sectioned at $-25^{\circ} \mathrm{C}$ to produce OCT-free 20-micron-thick tissue flakes. Only areas of each histological slide with uniform histological results (either radiation necrosis or tumor) were submitted for metabolomic analysis. The research study described here was performed with the approval of Yale Pathology Tissue Services.

\section{Sample Preparation for GC-TOF}

For each sample, $4 \mathrm{mg}$ of tissue was weighed and added to $1.0 \mathrm{ml}$ of acetonitrile, isopropanol, and water (3:3:2). The samples were homogenized using Geno/Grinder and centrifuged at $2500 \mathrm{rpm}$ for 5 minutes. The samples were then evaporated in a Labconco CentriVap cold trap concentrator and resuspended in $500 \mu \mathrm{l}$ of $50 \%$ acetonitrile. After being centrifuged for 2 minutes at 14,000 relative centrifugal force, the supernatant was removed, evaporated, and submitted for 2-step derivatization with methoxyamine in pyridine followed by sialylation with $N$-Methyl$N$-(trimethylsilyl) trifluoroacetamide (MSTFA).

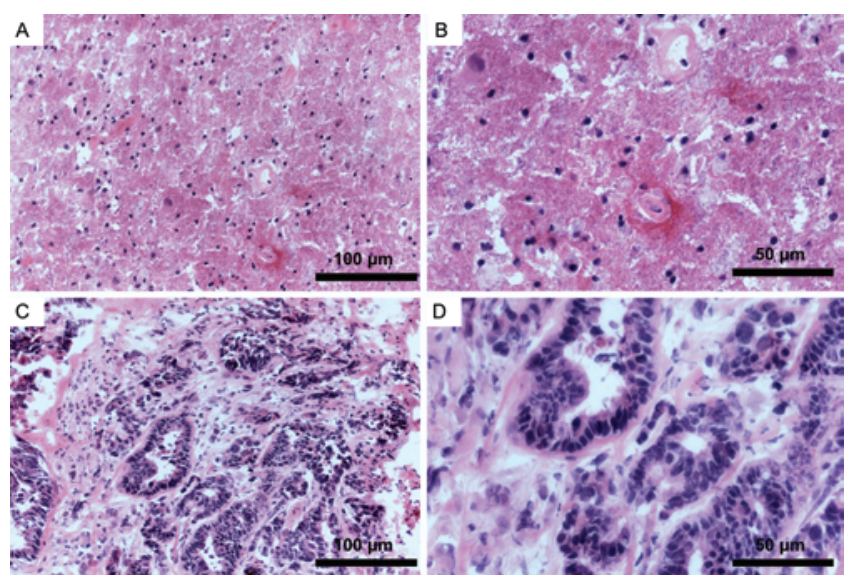

FIG. 1. Histological identification of radiation necrosis and tumor in sections stained with $\mathrm{H} \& \mathrm{E}$. The identity of each flash-frozen sample was confirmed histologically. A and B: Photomicrographs showing radiation necrosis, which was defined as inflammatory demyelination and reactive gliosis in the absence of tumor cells. C and D: Photomicrographs showing recurrent NSCLC. Metastatic tumors from various primary tumors were also identified histologically. Figure is available in color online only. 


\section{Methodology for GC-TOF}

The methodologies used were described in a previous study. ${ }^{5}$ An Agilent 6890 Gas Chromatograph was equipped with a GERSTEL automatic liner exchange system and a GERSTEL cold injection system with a temperature program as follows: $50^{\circ} \mathrm{C}$ to $275^{\circ} \mathrm{C}$ final temperature at a rate of $12^{\circ} \mathrm{C} / \mathrm{sec}$ and hold for 3 minutes. The injection volume was $0.5 \mu \mathrm{l}$ with a $10 \mu \mathrm{l} / \mathrm{sec}$ injection speed on a splitless injector with a purge time of 25 seconds.

The gas chromatograph separation column consisted of a 30-m long, 0.25-mm Rtx-5Sil mass spectroscopy column with a $0.25-\mu \mathrm{m} 95 \%$ dimethyl $/ 5 \%$ diphenyl polysiloxane film and an additional $10-\mathrm{m}$ integrated guard column. For the separation parameters, $99.9999 \%$ ultra-pure helium with built-in purifier was used as a carrier gas at a constant flow of $1 \mathrm{ml} /$ minute with the oven temperature held constant at $50^{\circ} \mathrm{C}$ for 1 minute and then increased by $20^{\circ} \mathrm{C}$ per minute to $330^{\circ} \mathrm{C}$, which was held constant for 5 minutes.

A Leco Pegasus IV TOF mass spectrometer controlled by Leco ChromaTOF software was used. The samples were introduced with a transfer line temperature set at $280^{\circ} \mathrm{C}$. Electron impact ionization occurred at $70 \mathrm{eV}$ with an ion source temperature of $250^{\circ} \mathrm{C}$. Two blank samples and 4 calibrates were included in each run for quality control.

\section{Data Processing}

Gas chromatography/mass spectroscopy peaks were annotated only if identified according to Metabolomics Standards Initiative guidelines, with both mass spectra and retention index recorded and matched. All signals were exported by the BinBase database and were reported by quantification ion, a unique database identifier, retention index, and the complete mass spectrum encoded as string.

Result files were transformed by calculating the sum of all structurally identified compounds for each sample and by dividing all data associated with a sample by the corresponding metabolite sum. These data were multiplied by a constant factor to obtain values without decimal places; intensities of identified metabolites with more than 1 peak were summed to only 1 value in the transformed data set.

\section{Statistical Analysis}

Statistical testing was conducted using $\mathrm{R}$ version 3.2.3, a modular open-source programming suite (http:// cran.r-project.org/). A principal component analysis was performed to check for discrimination accuracy between comparison groups using the ropls $\mathrm{R}$ package. A random forest analysis using 10,000 trees was performed to obtain predictive accuracy metrics and a ranked list of metabolites based on importance to the classification scheme. The random forest analysis was conducted using the randomForest R package.

Univariate statistical analysis included the MannWhitney U-test and fold change between the comparison groups. Positive fold-change values indicate elevated levels in radiation necrosis, whereas negative fold-change values indicate elevated levels in tumor. Statistical significance was defined as $p<0.05$ and fold change $>1.5$. An unsu- pervised hierarchical clustering analysis was performed to obtain metabolomic subtypes, conducted using the heatmap.plus R package. All data visualization was performed with the ggplot $2 \mathrm{R}$ package.

\section{Results}

\section{Patient Demographic Data and Pathological Tissue Characteristics}

Patient demographic data and pathological characteristics of specimens are summarized in Table 1. The average age of patients at the time of biopsy was 53.9 years. Two patients $(20 \%)$ were men, and $8(80 \%)$ were women. Of the 10 patients, 4 contributed only radiation necrosis specimens, and 3 contributed only tumor specimens; 3 patients contributed both radiation necrosis and tumor specimens (Table 2). Tumor samples consisted of 3 primary tumor types: melanoma, breast, and non-small cell lung cancer (NSCLC).

\section{Global Metabolite Analysis}

Our protocol applied GC-TOF for pathologically confirmed radiation necrosis and recurrent tumor samples. In total, we identified 141 metabolites that could be confidently mapped to known biochemical structures. A principal component analysis of the global metabolite profiles suggested high discrimination accuracy between the comparison groups, as shown in Fig. 2 upper. The variances of the first and second principal component were $43.83 \%$ and $24.86 \%$, respectively.

A random forest analysis yielded an overall predictive accuracy of $78.57 \%$. The random forest analysis also produced a ranked list of metabolites to distinguish the comparison groups. The top 25 metabolites are shown in Fig. 2 lower.

\section{Univariate Statistical Analysis of Identified Metabolites}

Of the 25 metabolites identified with the random forest analysis, 17 metabolites had levels that were significantly different between radiation necrosis and tumor samples ( $p<0.05$ by Mann-Whitney U-test, as shown in Table 3). These metabolites included alpha-tocopherol, proline, citric acid, gamma-tocopherol, UDP-glucuronic acid, butyrolactam, 2,5-dihydroxypyrazine, arachidonic acid, elaidic acid, taurine, UDP- $N$-acetylglucosamine, ribitol, adenosine-5-monophosphate, beta-sitosterol, conduritolbeta-epoxide, lauric acid, and putrescine. Of these metabolites, 6 were increased in tumor, and 11 were increased in radiation necrosis; all metabolites had an absolute fold change $>0.5$ between comparison groups.

\section{Metabolite Cluster Analysis}

An unsupervised hierarchical clustering analysis of the 17 metabolites identified showed 3 distinct clusters of metabolites that discriminate the comparison groups (Fig. 3). The 3 clusters represent increased metabolism (elevated in tumor), fatty acid products (elevated in radiation necrosis), and antioxidants/cofactors (elevated in radiation necrosis). The 2 metabolites that were most significantly elevated in radiation necrosis compared with tumor were alpha-to- 
TABLE 1. Patient demographic data and pathological characteristics of specimens

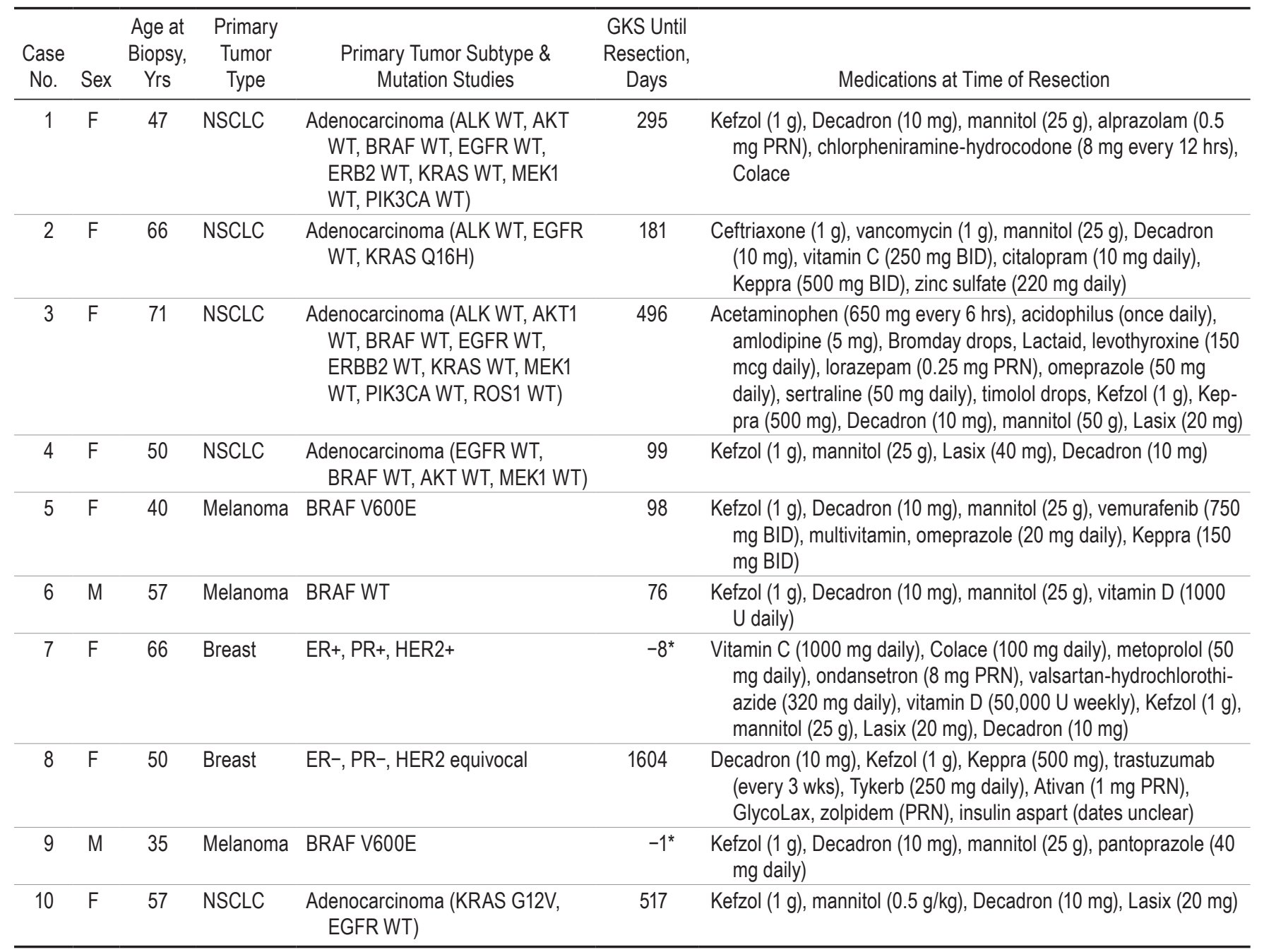

$\mathrm{BID}=$ twice daily; $\mathrm{PRN}=$ as necessary; $\mathrm{WT}=$ wild type.

* Two samples of tumor included for metabolomic analysis were not irradiated prior to resection; these patients underwent GKS after resection.

copherol and citric acid. The 2 metabolites that were most elevated in tumor compared with radiation necrosis were proline and UDP-glucuronic acid. Boxplots of the metabolite levels between comparison groups are shown in Fig. 4. The receiver operating characteristic (ROC) curves of these metabolites have area under the curve (AUC) values of 1.00 (alpha-tocopherol), 0.92 (citric acid), 0.94 (proline), and 0.93 (UDP-glucuronic acid).

\section{Analysis of Metabolites Currently Used in MRS and PET}

$\mathrm{N}$-acetylaspartate (NAA), along with creatine and choline, are the metabolites currently used in MRS for identification of radiation necrosis versus recurrent tumor. Of these 3 metabolites, only NAA was identified with the present study's methodologies. NAA trended toward but was not found to be significantly elevated in radiation necrosis compared with tumor $(\mathrm{p}=0.073$; fold change $=$ 1.36). The ROC curve of NAA has an AUC value of 0.8 .

In PET, analogs of glucose are most commonly used, although amino acids such as methionine, phenylalanine, and tyrosine have been studied at a few dedicated centers. Glucose and the individual amino acids were identified with the present study's methodologies. The first quartile, median, and third quartile values of glucose trended to be higher in tumor compared with radiation necrosis, but these results did not reach statistical significance ( $\mathrm{p}$ $=0.53$ ). Methionine, phenylalanine, and tyrosine also trended to be elevated in tumor compared with radiation necrosis, but again did not reach statistical significance $(\mathrm{p}=0.62,0.71$, and 0.80 ; fold change $=-0.17,-0.36$, and -0.13 , respectively). The ROC curves of glucose, methionine, phenylalanine, and tyrosine had AUC values of 0.61 , $0.59,0.55$, and 0.57 , respectively.

\section{Discussion}

A noninvasive method to differentiate radiation necrosis from recurrent tumor remains elusive. However, such a method is much needed today as the clinical use of ra- 

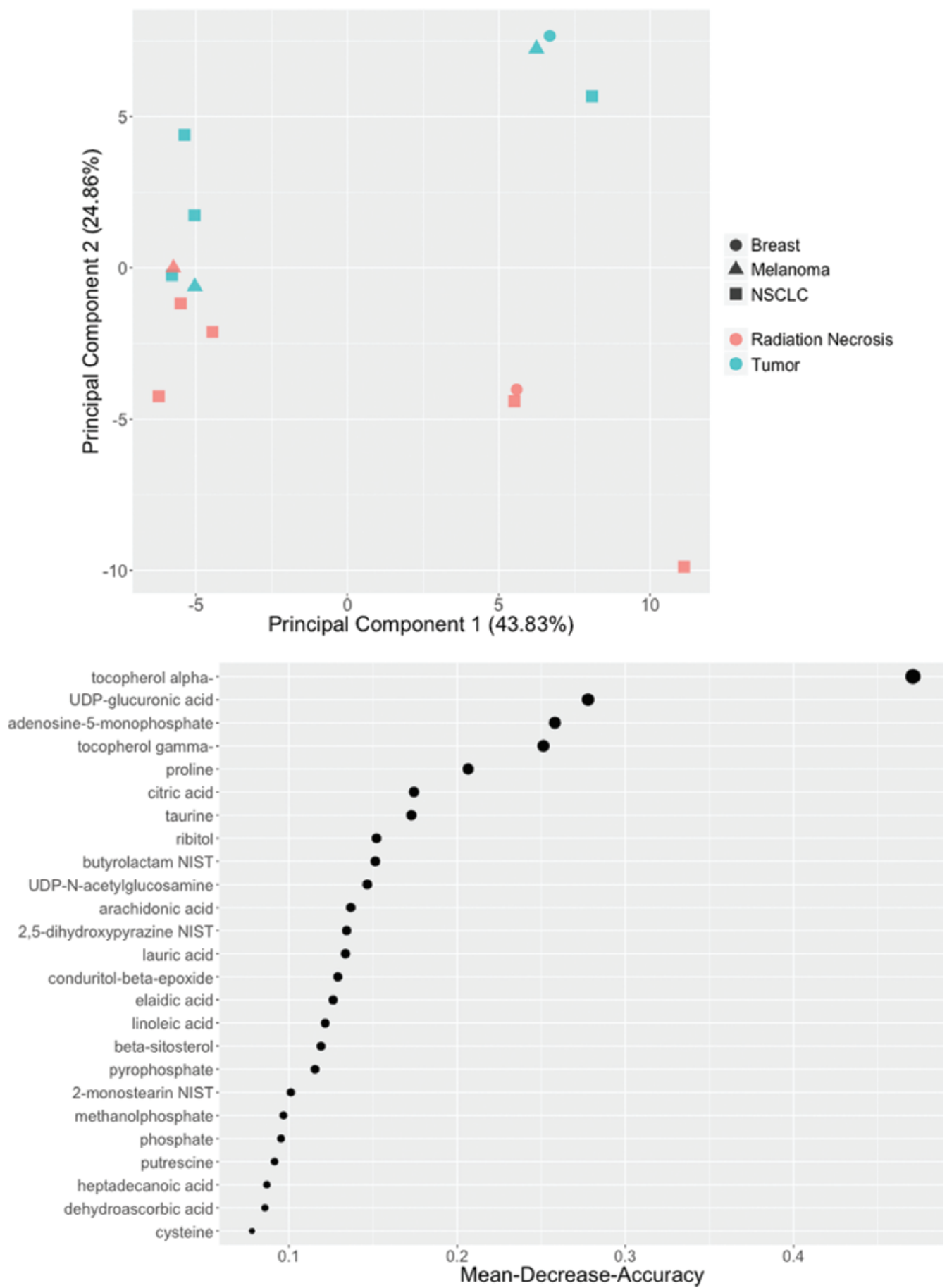

FIG. 2. Principal component analysis plots and random forest analysis show high discrimination accuracy between radiation necrosis and tumor. Upper: Principal component analysis score plot showing differential variance between tumor and radiation necrosis as well as primary tumor types. Lower: Random forest analysis identifies a ranked list of metabolites for classification of tumor versus radiation necrosis. NIST = National Institute of Standards and Technology. Figure is available in color online only.

diosurgery increases ${ }^{11}$ and patient survival continues to lengthen. ${ }^{14}$ This study aimed to examine currently available imaging metabolites to validate their use specifically in differentiating radiation necrosis and metastatic tumor. In addition, this study aimed to identify novel metabolites that may serve as alternative in vivo biomarkers for imaging.
When using in vivo metabolite biomarkers, MRS and PET should be the most promising imaging modalities. Current MRS methods examine choline/creatine and choline/NAA ratios. In the present study, we were only able to measure NAA; therefore, we were not able to make conclusions about the validity of using these ratios.

Current PET radiotracer analogs include glucose, me- 
TABLE 2. Description of analyzed specimens by primary tumor type and subsequent pathology

\begin{tabular}{rrrll}
\hline $\begin{array}{c}\text { Specimen } \\
\text { No. }\end{array}$ & $\begin{array}{c}\text { Case } \\
\text { No. }\end{array}$ & $\begin{array}{c}\text { Primary } \\
\text { Tumor } \\
\text { Type }\end{array}$ & \multicolumn{1}{c}{ Pathology } & \multicolumn{1}{c}{$\begin{array}{c}\text { Biopsy } \\
\text { Location }\end{array}$} \\
\hline 1 & 1 & NSCLC & Recurrent tumor & Frontal \\
\hline 2 & 1 & NSCLC & Radiation necrosis & Frontal \\
\hline 3 & 2 & NSCLC & Recurrent tumor & Frontal \\
\hline 4 & 2 & NSCLC & Radiation necrosis & Frontal \\
\hline 5 & 3 & NSCLC & Recurrent tumor & Frontoparietal \\
\hline 6 & 3 & NSCLC & Radiation necrosis & Frontoparietal \\
\hline 7 & 3 & NSCLC & Recurrent tumor & Frontoparietal \\
\hline 8 & 4 & NSCLC & Radiation necrosis & Parietal \\
\hline 9 & 5 & Melanoma & Recurrent tumor & Frontal \\
\hline 10 & 6 & Melanoma & Radiation necrosis & Frontal \\
\hline 11 & 7 & Breast & Metastatic tumor & Cerebellar \\
\hline 12 & 8 & Breast & Radiation necrosis & Frontoparietal \\
\hline 13 & 9 & Melanoma & Metastatic tumor & Rt frontal \\
\hline 14 & 10 & NSCLC & Radiation necrosis & Temporal \\
\hline
\end{tabular}

thionine, phenylalanine, and tyrosine in the form of FDG, L-methyl- ${ }^{11} \mathrm{C}$-methionine $\left({ }^{11} \mathrm{C}-\mathrm{MET}\right), 3,4$-dihydroxy-6- ${ }^{18} \mathrm{~F}-$ fluoro-L-phenylalanine ( $\left.{ }^{18} \mathrm{~F}-\mathrm{FDOPA}\right)$, and $\mathrm{O}-2-{ }^{18} \mathrm{~F}-$-fluoroethyl-L-tyrosine $\left({ }^{18} \mathrm{~F}-\mathrm{FET}\right)$, respectively. For all of these metabolites, the underlying assumption is that tumor is more metabolically active than radiation necrosis and accumulates higher levels of these metabolites. FDG-PET has been the most studied but suffers from wide ranges of reported sensitivities $(65 \%-81 \%)$ and specificities $(40 \%-$ $94 \%) .{ }^{16}$ Our results found that these 4 metabolites trended
TABLE 3. Significant changes in metabolites between radiation necrosis and tumor samples

\begin{tabular}{llrc}
\hline \multicolumn{1}{c}{ Metabolite } & \multicolumn{1}{c}{ KEGG } & $\begin{array}{c}\text { Fold } \\
\text { Change }\end{array}$ & $\begin{array}{c}\text { Nominal } \\
\text { p Value }\end{array}$ \\
\hline Alpha-tocopherol & C02477 & 2.91 & $5.83 \mathrm{E}-04$ \\
\hline Proline & $\mathrm{C} 00148$ & -3.04 & $4.08 \mathrm{E}-03$ \\
\hline Citric acid & $\mathrm{C} 00158$ & 1.15 & $6.99 \mathrm{E}-03$ \\
\hline Gamma-tocopherol & $\mathrm{C} 02483$ & 2.75 & $6.99 \mathrm{E}-03$ \\
\hline UDP-glucuronic acid & $\mathrm{C} 00167$ & -3.81 & $8.66 \mathrm{E}-03$ \\
\hline Butyrolactam & $\mathrm{C} 11118$ & 5.89 & $1.11 \mathrm{E}-02$ \\
\hline 2,5-Dihydroxypyrazine & $\mathrm{NA}$ & -2.05 & $1.75 \mathrm{E}-02$ \\
\hline Arachidonic acid & $\mathrm{C} 00219$ & 1.63 & $1.75 \mathrm{E}-02$ \\
\hline Elaidic acid & $\mathrm{C} 00712$ & 0.86 & $1.75 \mathrm{E}-02$ \\
\hline Taurine & $\mathrm{C} 00245$ & -2.21 & $1.75 \mathrm{E}-02$ \\
\hline UDP-N-acetylglucosamine & $\mathrm{C} 00043$ & -2.11 & $1.75 \mathrm{E}-02$ \\
\hline Ribitol & $\mathrm{C} 00474$ & 1.94 & $2.13 \mathrm{E}-02$ \\
\hline Adenosine-5-monophosphate & $\mathrm{C} 00020$ & -0.87 & $2.62 \mathrm{E}-02$ \\
\hline Beta-sitosterol & $\mathrm{C} 01753$ & 1.18 & $2.62 \mathrm{E}-02$ \\
\hline Conduritol-beta-epoxide & $\mathrm{NA}$ & 2.13 & $3.79 \mathrm{E}-02$ \\
\hline Lauric acid & $\mathrm{C} 02679$ & 0.91 & $3.79 \mathrm{E}-02$ \\
\hline Putrescine & $\mathrm{C} 00134$ & 1.47 & $3.79 \mathrm{E}-02$ \\
\hline KEGGID Ky & &
\end{tabular}

KEGG ID = Kyoto Encyclopedia of Genes and Genomes identifier; NA = not available.

to be elevated in tumor compared with radiation necrosis, supporting the currently used PET methodology. However, the ability to discriminate between radiation necrosis and tumor appeared to be low compared with that of other candidate metabolites in our study.

We identified 17 novel metabolites that may have higher

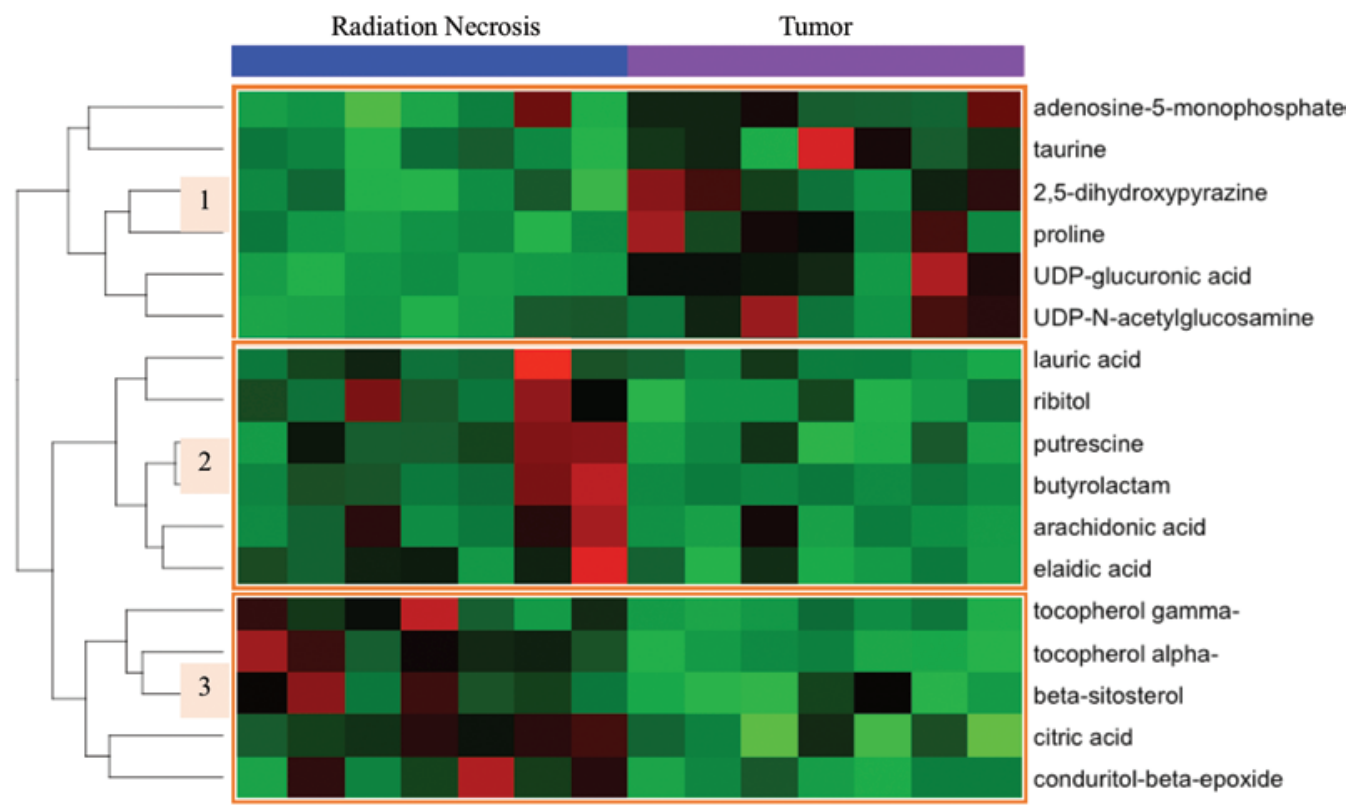

FIG. 3. Heat map of significant metabolites reveals metabolic signatures of radiation necrosis and tumor. An unsupervised hierarchical clustering analysis shows 3 distinct clusters of metabolites that discriminate between radiation necrosis and tumor. Metabolites that represent increased metabolism were elevated in tumor. Metabolites that represent fatty acid products and antioxidants/ cofactors were elevated in radiation necrosis. Figure is available in color online only. 

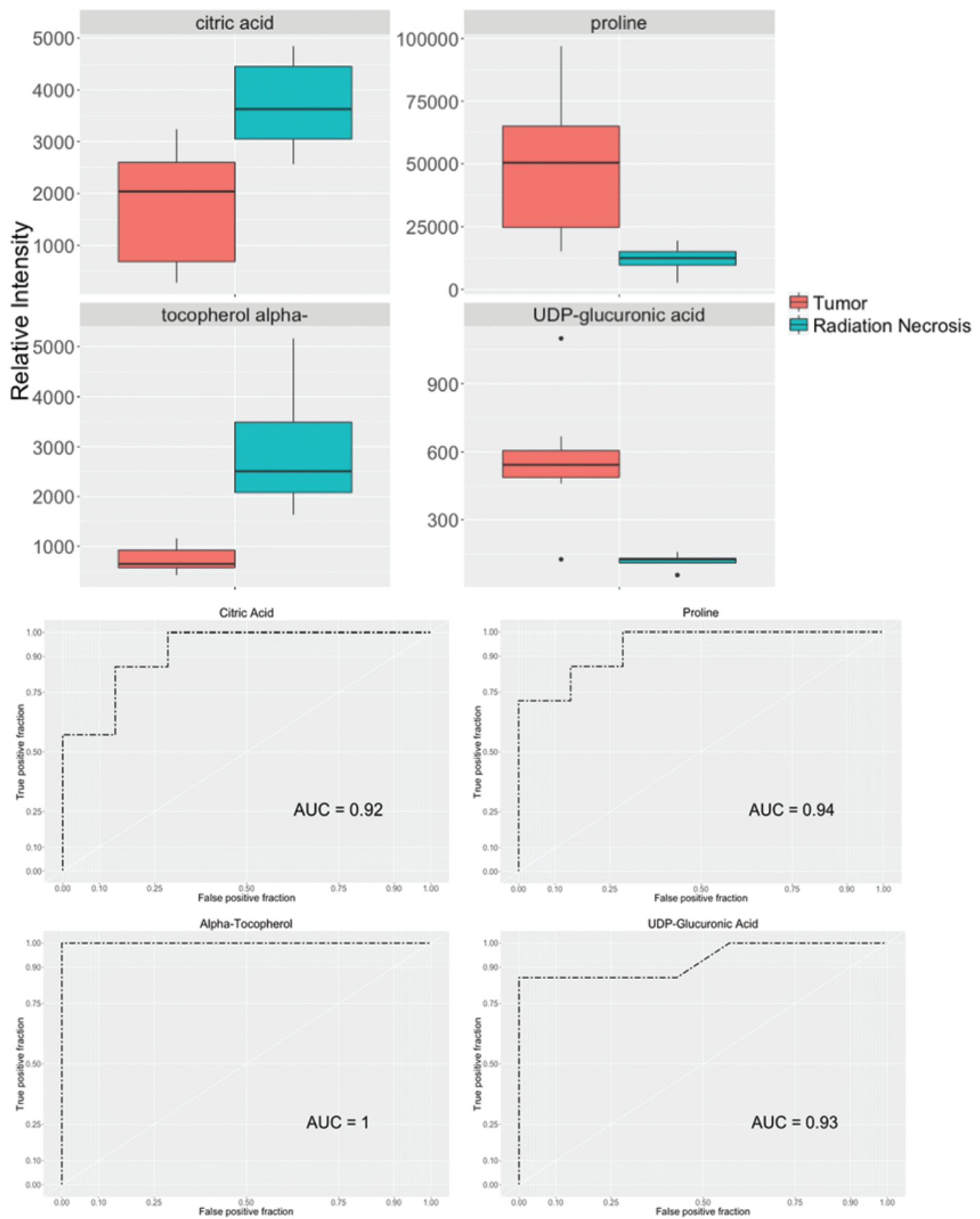

FIG. 4. Boxplots (upper) and ROC curves (lower) for selected metabolites discriminating between tumor and radiation necrosis. Selected metabolites provide a high degree of discrimination between tumor and radiation necrosis according to the AUC values of the ROC curves. Figure is available in color online only.

diagnostic potential if they can be translated into imaging studies. Our results suggest that radiation necrosis had elevated levels of fatty acids and antioxidant metabolites, whereas tumor had elevated markers of energy metabolism.

In radiation necrosis, demyelination and cell death may result in increased fatty acid products; we identified lauric acid, ribitol, putrescine, butyrolactam, arachidonic acid, and elaidic acid as potential biomarkers in this group. Previous studies have found that oligodendrocytes are extremely sensitive to radiation. ${ }^{2,3}$ In addition, MRS of radiation necrosis often contains a large lipid peak, but no studies have resolved the identity of the involved metabolites. ${ }^{2,6,13}$ The biomarkers identified in this study may contribute to this peak.

In addition, radiation necrosis tissue may mobilize elevated levels of antioxidants in response to oxidative stress and increased cell death. Our study identified gamma- 
tocopherol, alpha-tocopherol, beta-sitosterol, citric acid, and conduritol-beta-epoxide as candidate biomarkers. Although it is uncertain why these metabolites were elevated in radiation necrosis, one explanation could be bias due to unreported patient oral supplementation. However, given that 3 patients contributed both tumor and radiation necrosis samples, the likelihood of this bias is low.

Tumor is more anabolically active than normal tissue and is probably more active than radiation necrosis; we found that tumor had increased levels of adenosine-5-monophosphate, taurine, 2,5-dihydroxypyrazine, proline, UDP-glucuronic acid, and UDP- $N$-acetylglucosamine. Taurine has been shown to be elevated in samples of lung cancer tissue compared with paracarcinomatous tissue..$^{19}$ In addition, proline biosynthesis has been shown to augment tumor cell growth and aerobic glycolysis. ${ }^{8}$ UDP-glucuronic acid and UDP- $N$-acetylglucosamine are both used by glucuronosyltransferase and glycosyltransferase reactions. Using UDP$\mathrm{N}$-acetylglucosamine as a substrate, multiple tumor types (including breast, prostate, lung, liver, colon, and bladder cancers) have increased O-linked- $\beta$ - $N$-acetylglucosamine modifications on intracellular proteins through the mTOR/ MYC pathway. ${ }^{15}$ Elevated levels of UDP- $N$-acetylglucosamine have also been found in human lung adenocarcinoma compared with nonmalignant tissue, ${ }^{18}$ as well as in human melanoma cells implanted in mice. ${ }^{4}$

There are several limitations to this study. First, due to the low number of available tissue samples, no metabolites in the univariate statistical analysis reached significance with Bonferroni correction (significance threshold $\mathrm{p}=3.54 \mathrm{E}-4)$. This study was originally performed as a proof-of-concept study to determine if unique metabolites could be identified for radiation necrosis versus tumor. Although several metabolites have been identified, the validity of our results still needs to be confirmed by further studies that include a larger sample size and a larger variety of metastatic tumors. In addition, there are no data from this study to suggest that similar metabolites should be used to differentiate primary brain tumors from radiation necrosis. Second, this study does not provide any correlation with currently available imaging changes. Third, this study does not address possible metabolite changes in pseudo-progression because it remains unclear if the pathophysiology of early postradiation changes (such as pseudo-progression) is the same as the delayed changes of radiation necrosis.

We acknowledge that these data are preliminary and that future work must be done to validate these metabolites in combination with the development of in vivo neuroimaging modalities. If noninvasive imaging modalities can be developed specifically for the metabolites identified in this study, however, this may be the first step to robustly solving the current dilemma of distinguishing radiation necrosis and recurrent tumor after radiosurgery.

\section{Conclusions}

To our knowledge, this study is the first tissue-based, untargeted metabolomics analysis to examine differential metabolic profiles of radiation necrosis versus tumor. We identified multiple candidate metabolites that may be used with imaging modalities such as MRS and PET for differentiating radiation necrosis and tumor; these candidate metabolites may have much higher binary classification test results compared with those of currently used metabolites in MRS and PET. Although promising, a prospective study using the identified metabolites with radiographic techniques is needed to test the applicability of these results.

\section{Acknowledgments}

We thank the West Coast Metabolomics Center and Mine Palazoglu for assistance with GC-TOF and the Methods.

\section{References}

1. Alomari A, Rauch PJ, Orsaria M, Minja FJ, Chiang VL, Vortmeyer AO: Radiologic and histologic consequences of radiosurgery for brain tumors. J Neurooncol 117:33-42, 2014

2. Burger PC, Boyko OB: The pathology of central nervous system radiation injury, in Gutin PH, Leibel SA, Sheline GE (eds): Radiation Injury to the Nervous System. NY: Raven, 1991, pp 191-208

3. Castel JC, Caillé JM: Imaging of irradiated brain tumours. Value of magnetic resonance imaging. J Neuroradiol 16:81132,1989

4. Corbett RJ, Nunnally RL, Giovanella BC, Antich PP: Characterization of the 31P nuclear magnetic resonance spectrum from human melanoma tumors implanted in nude mice. Cancer Res 47:5065-5069, 1987

5. Fiehn O, Wohlgemuth G, Scholz M, Kind T, Lee DY, Lu Y, et al: Quality control for plant metabolomics: reporting MSIcompliant studies. Plant J 53:691-704, 2008

6. Kimura T, Sako K, Gotoh T, Tanaka K, Tanaka T: In vivo single-voxel proton MR spectroscopy in brain lesions with ring-like enhancement. NMR Biomed 14:339-349, 2001

7. Kind T, Tolstikov V, Fiehn O, Weiss RH: A comprehensive urinary metabolomic approach for identifying kidney cancer. Anal Biochem 363:185-195, 2007

8. Liu W, Hancock CN, Fischer JW, Harman M, Phang JM: Proline biosynthesis augments tumor cell growth and aerobic glycolysis: involvement of pyridine nucleotides. Sci Rep 5:17206, 2015

9. Nath SK, Sheridan AD, Rauch PJ, Yu JB, Minja FJ, Vortmeyer AO, et al: Significance of histology in determining management of lesions regrowing after radiosurgery. $\mathbf{J}$ Neurooncol 117:303-310, 2014

10. Nishiumi S, Kobayashi T, Ikeda A, Yoshie T, Kibi M, Izumi $\mathrm{Y}$, et al: A novel serum metabolomics-based diagnostic approach for colorectal cancer. PLoS One 7:e40459, 2012

11. Park HS, Wang EH, Rutter CE, Corso CD, Chiang VL, Yu JB: Changing practice patterns of Gamma Knife versus linear accelerator-based stereotactic radiosurgery for brain metastases in the US. J Neurosurg 124:1018-1024, 2016

12. Rauch PJ, Park HS, Knisely JP, Chiang VL, Vortmeyer AO: Delayed radiation-induced vasculitic leukoencephalopathy. Int J Radiat Oncol Biol Phys 83:369-375, 2012

13. Shah R, Vattoth S, Jacob R, Manzil FF, O’Malley JP, Borghei $\mathrm{P}$, et al: Radiation necrosis in the brain: imaging features and differentiation from tumor recurrence. Radiographics 32:1343-1359, 2012

14. Sneed PK, Mendez J, Vemer-van den Hoek JG, Seymour ZA, Ma L, Molinaro AM, et al: Adverse radiation effect after stereotactic radiosurgery for brain metastases: incidence, time course, and risk factors. J Neurosurg 123:373-386, 2015

15. Sodi VL, Khaku S, Krutilina R, Schwab LP, Vocadlo DJ, 
Seagroves TN, et al: mTOR/MYC axis regulates O-GlcNAc transferase expression and O-GlcNAcylation in breast cancer. Mol Cancer Res 13:923-933, 2015

16. Verma N, Cowperthwaite MC, Burnett MG, Markey MK: Differentiating tumor recurrence from treatment necrosis: a review of neuro-oncologic imaging strategies. Neuro Oncol 15:515-534, 2013

17. Wibom C, Surowiec I, Mörén L, Bergström P, Johansson M, Antti $\mathrm{H}$, et al: Metabolomic patterns in glioblastoma and changes during radiotherapy: a clinical microdialysis study. J Proteome Res 9:2909-2919, 2010

18. Wikoff WR, Grapov D, Fahrmann JF, DeFelice B, Rom WN, Pass HI, et al: Metabolomic markers of altered nucleotide metabolism in early stage adenocarcinoma. Cancer Prev Res (Phila) 8:410-418, 2015

19. Zhao Q, Cao Y, Wang Y, Hu C, Hu A, Ruan L, et al: Plasma and tissue free amino acid profiles and their concentration correlation in patients with lung cancer. Asia Pac J Clin Nutr 23:429-436, 2014

\section{Disclosures}

The authors report no conflict of interest concerning the materials or methods used in this study or the findings specified in this paper.

\section{Author Contributions}

Conception and design: Chiang, Lu, Turban, Damisah, Li, Eid, Vortmeyer. Acquisition of data: Lu, Turban, Li, Alomari, Vortmeyer. Analysis and interpretation of data: Lu, Turban, Vortmeyer. Drafting the article: Chiang, Lu, Turban. Critically revising the article: Chiang, Lu, Turban, Vortmeyer. Reviewed submitted version of manuscript: Chiang, Lu, Turban. Approved the final version of the manuscript on behalf of all authors: Chiang. Statistical analysis: Lu. Administrative/technical/material support: Chiang, Lu, Turban, Damisah, Eid, Vortmeyer. Study supervision: Chiang, Damisah, Eid, Vortmeyer.

\section{Supplemental Information}

Previous Presentations

Portions of this work were presented as a flash oral presentation at the 17th Annual Leksell Conference, New York, NY, May 12, 2014.

\section{Correspondence}

Veronica Chiang, Yale Department of Neurosurgery, PO Box 208082, New Haven, CT 06520-8082. email: veronica.chiang@ yale.edu. 